Series A

I. MATHEMATICA

412

\title{
ZUR CHARAKTERISIERUNG HARMONISCHER UND SUBHARMONISCHER FUNKTIONEN DURCH MITTELUNGSPROZESSE
}

\author{
VON \\ ALEXANDER DINGHAS \\ Herrn Professor P. J. Myrberg zum 75. Geburtstag gewidmet
}


Am 12. Mai 1967 vorgelegt von Rolf Nevanlinna und Oldi Lehto 


\section{Zur Charakterisierung harmonischer und subharmonischer Funktionen durch Mittelungsprozesse}

1. Einleitung. Es bedeute $X$ einen Raum und $A[X]$ einen auf $X$ definierten Funktionenraum. Wir bezeichnen mit $x$ die Elemente von $X$ und $f=[f(x)] \quad(x \in X)$ die Elemente von $A[X]$ und nennen $f(x)$ die Komponenten von $f$. Es wird grundsätzlich angenommen, dass die durch $f$ vermittelte Abbildung von $X$ reellwertig ist, d.h. dass der Rang von $f$ in $\boldsymbol{R}$ bzw. ${ }^{\wedge} \boldsymbol{R}$ enthalten ist. Dabei soll hier unter $\widehat{\wedge}$ die nach links durch den Punkt $-\infty$ kompaktifizierte reelle Zahlengerade bedeuten.

Ist $A[X]$ ein linearer Raum und die Abbildung

$$
T: A[X] \rightarrow \wedge \boldsymbol{R}
$$

im Sinne der Gleichungen

$$
T\left(f_{1}+f_{2}\right)=T f_{1}+T f_{2} \quad\left(f_{1}, f_{2} \in A[X]\right)
$$

und (für $\lambda \in R$ )

$$
T(\lambda f)=\lambda T f
$$

linear, so wird $T$, wie üblich, als linearer Operator bezeichnet.

Es seien jetzt $T_{1}, T_{2}$ zwei auf $A[X]$ gegebene Operatoren und $Y_{1}, Y_{2}$ die durch die Gleichungen

$$
Y_{1}=\left\{f \mid f \in A[X], T_{1} f=0\right\}
$$

bzw.

$$
Y_{2}=\left\{f \mid f \in A[X], T_{2} f=0\right\}
$$

definierten (nach Voraussetzung nicht leeren) Teilmengen von $A[X]$. Dann sollen die Operatoren $T_{1}, T_{2}$ als äquivalent bezeichnet werden, wenn $Y_{1}=Y_{2}$ ist. Eine ähnliche Bezeichnung soll gelten, wenn man in (1.4) und (1.5) die Bedingungen $T_{1} f=0$ bzw. $T_{2} f=0$ durch $T_{1} f \geqq 0$ bzw. $T_{2} f \geqq 0$ ersetzt. In der vorliegenden Note wird $X$ eine feste offene Teilmenge $O$ des $n$-dimensionalen euklidischen Raumes $\boldsymbol{R}^{\boldsymbol{n}}$ ( $n \geqq 1$ ) bedeuten, und $A[O]$ eine geeignet gewählte Menge reellwertiger, insbesondere harmonischer und subharmonischer Funktionen. Gegenstand 
der vorliegenden Arbeit sollen zunächst Äquivalenzfragen zwischen den klassischen Operatoren von Laplace und Blaschke-Privaloff sowie eines allgemeineren Operators, der nach H. A. Schwarz benannt wird, bilden ${ }^{\mathbf{1}}$ ). Die letzten Nummern der Arbeit befassen sich mit entsprechenden Fragen in Riemannschen Räumen sowie mit Anwendungen auf einige einfache Klassen elliptischer partieller Differentialgleichungen.

2. Vorbereitende Tatsachen. Im folgenden soll $x=\left(x_{1}, \ldots, x_{n}\right)$ einen Punkt von $\boldsymbol{R}^{n}$ bedeuten, und $\|x\|$ dessen Norm $\left(x_{1}^{2}+\ldots+x_{n}^{2}\right)^{1 / 2}$. Ferner soll bei gegebenen Vektoren $\mathfrak{l}_{1}, \ldots, \mathfrak{h}_{m}(m \geqq 1)$ von $\boldsymbol{R}^{n}$ mit dem Anfangspunkt in $0 \quad H_{x}=\left(\mathfrak{h}_{1}, \ldots, \mathfrak{h}_{m}\right)$ das entsprechende lokale Koordinatensystem des durch $\mathfrak{h}_{1}, \ldots, \mathfrak{h}_{m}$ definierten linearen Unterraumes bedeuten. Danach gehen die Systeme $H_{x}=\left(\mathfrak{h}_{1}, \ldots, \mathfrak{h}_{m}\right)$ und $H_{y}=\left(\mathfrak{h}_{1}, \ldots, \mathfrak{h}_{m}\right)$ durch Translation auseinander hervor.

Nachfolgende Sätze, welche die Existenz bestimmter Klassen von Vektorsystemen mit vorgeschriebenen Symmetrieeigenschaften sichern, sind für die Entwicklungen dieser Arbeit von grundlegender Bedeutung:

Hilfssatz 1. Es sei $n \geqq 1$ ganz. Dann hat das Gleichungssystem

$$
\begin{aligned}
& \sum_{\mu=1}^{n} x_{i \mu}^{2}=1 \quad(i=1, \ldots, n+1), \\
& \sum_{\mu=1}^{n} x_{i \mu} x_{k \mu}=-\frac{1}{n} \quad(i \neq k ; i, k=1, \ldots, n+1), \\
& \sum_{k=1}^{n+1} x_{k \mu}=0 \quad(\mu=1, \ldots, n) \\
& \frac{n}{n+1} \sum_{k=1}^{n+1} x_{k \mu} x_{k v}=\delta_{\mu \nu}=\left\{\begin{array}{ll}
1 & (\mu=v) \\
0 & (\mu \neq v)
\end{array}(\mu, v=1, \ldots, n)\right.
\end{aligned}
$$

stets eine reelle Lösung.

Beweis. Da für $n=1, x_{11}=1, x_{21}=-1$ genommen werden kann, so darf man ohne weiteres $n>1$ annehmen.

Man nehme nun an, dass die (reellen) Elemente $y_{k \sigma}$ der Matrix $\left(y_{k \sigma}\right)$ $(k=1, \ldots, n ; \sigma=1, \ldots, n-1)$ dem Gleichungssystem (2.1) mit $n-1$ anstelle von $n$ genügen. Setzt man dann

$$
\begin{aligned}
& x_{k \sigma}=\left(\frac{n^{2}-1}{n^{2}}\right)^{1 / 2} y_{k \sigma} \quad\left(\begin{array}{l}
k=1, \ldots n \\
\sigma=1, \ldots, n-1
\end{array}\right) \\
& x_{k n}=-\frac{1}{n} \quad(k=1, \ldots, n) \\
& x_{(n+1) k}=0 \\
& (k=1, \ldots, n-1)
\end{aligned}
$$

1) Man vgl. [1], S. $17 \mathrm{ff}$. 
und $x_{(n+1) n}=1$, so genügen die $x_{k \mu}$, wie man ohne weiteres nachprüft, dem Gleichungssystem (2.1).

Allgemein liefert die Ausgangsmatrix

$$
A_{1}=\left(\begin{array}{c}
1 \\
-1
\end{array}\right)
$$

durch Induktion die Matrizen

$$
A_{n}=\left(\begin{array}{cr}
\lambda_{n-1} A_{n-1} & -\frac{1}{n} \\
0 & 1
\end{array}\right) \quad(n=2,3, \ldots)
$$

mit $\lambda_{n-1}^{-1}=\left(n^{2} /\left(n^{2}-1\right)\right)^{1 / 2}$. Dabei bedeutet die rechte Seite eine Matrix mit $n+1$ Zeilen und $n$ Spalten, und zwar so, dass die mit $\lambda_{n-1}^{-1}$ multiplizierte Matrix $A_{n-1}$ von $\operatorname{der}((n+1)$-ten) Zeile $0,0, \ldots, 0,1$ und der (n-ten) Spalte $-1 / n,-1 / n, \ldots,-1 / n, 1$ umrahmt wird. Der Leser kann feststellen, dass

$$
A_{2}=\left(\begin{array}{rr}
\frac{\sqrt{3}}{2} & -\frac{1}{2} \\
-\frac{\sqrt{3}}{2} & -\frac{1}{2} \\
0 & 1
\end{array}\right)
$$

ist.

Bezeichnet man allgemein das innere Produkt zweier Vektoren $\mathfrak{a}, \mathfrak{b}$ (in dem dazugehörigen Raum) durch $\langle\mathfrak{a}, \mathfrak{b}\rangle$, so kann man den Inhalt des Hilfssatzes 1 in geometrischer Sprache auch folgendermassen formulieren:

Hilfssatz 2. Beigegebenem ganzzahligen $n \geqq 1$ existiert in $\boldsymbol{R}^{\boldsymbol{n}}$ ein Vektorsystem $\left(\mathfrak{a}_{1}, \ldots, \mathfrak{a}_{n+1}\right)$ mit den Eigenschaften:

1. Es gilt

$$
\left\langle\mathfrak{a}_{i}, \mathfrak{a}_{k}\right\rangle=\left\{\begin{array}{rr}
-\frac{1}{n} & (i \neq k) \\
1 & (i=k)
\end{array} \quad(i, k=1, \ldots, n+1) .\right.
$$

2. Man definiere mit Hilfe der Vektoren

$$
\mathfrak{a}_{k}=\left(a_{k 1}, \ldots, a_{k n}\right) \quad(k=1, \ldots, n+1)
$$


die Vektoren

$$
\mathfrak{a}_{i}^{\prime}=\left(a_{1 i}, \ldots, a_{(n+1) i}\right) \quad(i=1, \ldots, n) .
$$

Dann bildet das Vektorsystem $\left(\mathfrak{a}_{1}^{\prime}, \ldots, \mathfrak{a}_{n+1}^{\prime}\right)$, wobei

$$
\mathfrak{a}_{n+1}^{\prime}=\left[\mathfrak{a}_{1}^{\prime}, \ldots, \mathfrak{a}_{n}^{\prime}\right]
$$

ist, ein in $\boldsymbol{R}^{n+\mathbf{I}}$ orthogonales Vektorsystem.

Der Vektor $\left[\mathfrak{a}_{1}^{\prime}, \ldots, \mathfrak{a}_{n}^{\prime}\right]$ in $\boldsymbol{R}^{n+1}$ hat dieselbe Bedeutung wie das äussere Produkt $[\mathfrak{a}, \mathfrak{b}]$ (oder auch $\mathfrak{a} \wedge \mathfrak{b}$ ) in $\boldsymbol{R}^{\mathbf{3}}$ und wird durch den üblichen Prozess aus der Matrix

$$
M=\left(a_{k l}\right) \quad\left(\begin{array}{l}
k=1, \ldots, n+1 \\
l=1, \ldots, n
\end{array}\right)
$$

mit Hilfe der $n$ quadratischen Unterdeterminanten $n$-ter Ordnung (unter Einbeziehung der Orientierungsverhältnisse) konstruiert. Die Normierung des Systems $\left(\mathfrak{a}_{1}^{\prime}, \ldots, \mathfrak{a}_{n+1}^{\prime}\right)$ von $R^{n+1}$ wird durch folgenden Satz festgelegt:

Hilfssatz 3. Man setze

$$
\lambda^{2}=\frac{n}{n+1} \quad(\lambda>0),
$$

und bilde das Vektorsystem $\left(\mathfrak{c}_{1}, \ldots, \mathfrak{c}_{n+1}\right)$ mit

$$
\mathfrak{c}_{k}=\lambda \mathfrak{a}_{k}^{\prime} \quad(k=1, \ldots, n)
$$

und

$$
\mathfrak{c}_{n+1}=\left[\mathfrak{c}_{1}, \ldots, \mathfrak{c}_{n}\right]=\lambda^{n}\left[\mathfrak{a}_{1}, \ldots, \mathfrak{a}_{n}\right] .
$$

Dann ist $\left(\mathfrak{c}_{1}, \ldots, \mathfrak{c}_{n+1}\right)$ orthonormal in $\boldsymbol{R}^{n+1}$.

Beweis. Nach Definition ist

$$
\mathfrak{a}_{n+1}^{\prime}=\left(\Delta_{1}, \ldots, \Delta_{n+1}\right)
$$

wobei $(-1)^{k+1} \Delta_{k}$ gleich derjenigen Unterdeterminante ist, die man aus $M$ durch Streichung der $k$-ten Zeile erhält. Andererseits gilt nach einem klassischen Satz von Gram ${ }^{2}$ )

$$
\Delta_{k}^{2}=\left|\left\langle\mathfrak{a}_{\mu}, \mathfrak{a}_{v}\right\rangle\right| \quad(1 \leqq \mu, v \leqq n+1 ; \mu, v \neq k),
$$

wobei rechts die Determinante der in Frage kommenden inneren Produkte $\left\langle\mathfrak{a}_{i}, \mathfrak{a}_{k}\right\rangle$ bedeutet. Somit wird

2) Man vgl. etwa [3], S. 21. 


$$
\Delta_{k}^{2}=\left|\begin{array}{rrrrr}
1 & -\frac{1}{n} & \cdot & \cdot & -\frac{1}{n} \\
-\frac{1}{n} & 1 & \cdot & \cdot & -\frac{1}{n} \\
\cdot & \cdot & \cdot & \cdot \\
\cdot & \cdot & \cdot & -\frac{1}{n} \\
-\frac{1}{n} & -\frac{1}{n} & \cdot & -\frac{1}{n} & 1
\end{array}\right|=\left|\begin{array}{rrrrr}
\frac{1}{n} & \frac{1}{n} & \cdot & \cdot & \frac{1}{n} \\
-\frac{1}{n} & 1 & \cdot & -\frac{1}{n} \\
\cdot & \cdot & \cdot & \cdot \\
\cdot & \cdot & \cdot & -\frac{1}{n} \\
-\frac{1}{n} & -\frac{1}{n} & \cdot & -\frac{1}{n} & 1
\end{array}\right| .
$$

Man addiere jetzt in der letzten Determinante rechts die erste Zeile nacheinander zu der zweiten, dritten, ...,n-ten Zeile. Dann erhält man die Gleichung

$$
\Delta_{k}^{2}=\frac{1}{n+1}\left|\frac{n+1}{n} \delta_{\mu \nu}\right| \quad(\mu, v=1, \ldots, n)
$$

und somit

$$
\Delta_{k}^{2}=\frac{(n+1)^{n-1}}{n^{n}} \quad(k=1, \ldots, n) .
$$

Das liefert die Gleichung

$$
\left\|\mathfrak{a}_{n+1}^{\prime}\right\|^{2}=\left(\frac{n+1}{n}\right)^{n}
$$

d. h.

$$
\left[\mathfrak{c}_{1}, \ldots, \mathfrak{c}_{n}\right]^{2}=\left[\lambda \mathfrak{a}_{1}^{\prime}, \ldots, \lambda \mathfrak{a}_{n}^{\prime}\right]^{2}=\left(\frac{n}{n+1}\right)^{n}\left\|\mathfrak{a}_{n+1}^{\prime}\right\|^{2}=1
$$

Da nun andererseits mit Rücksicht auf Hilfssatz 1 (mit $a_{i k}$ anstelle $x_{i k}$ ) sowie auf die Definition von $\mathfrak{a}_{n+1}$ die Gleichungen

$$
\left\langle\mathfrak{c}_{i}, \mathfrak{c}_{k}\right\rangle=\lambda^{2}\left\langle\mathfrak{a}_{i}^{\prime}, \mathfrak{a}_{k}^{\prime}\right\rangle=\delta_{i k} \quad(i, k=1, \ldots, n)
$$

und

$$
\left\langle\mathfrak{c}_{k}, \mathfrak{c}_{n+1}\right\rangle=\lambda^{n+1}\left\langle\mathfrak{a}_{k}^{\prime},\left[\mathfrak{a}_{1}^{\prime}, \ldots, \mathfrak{a}_{n}^{\prime}\right]\right\rangle=0 \quad(k=1, \ldots, n)
$$

gelten, muss das Vektorsystem $\left(\mathfrak{c}_{1}, \ldots, \mathfrak{c}_{n+1}\right)$ orthonormal in $\boldsymbol{R}^{n+1}$ sein.

Da das Vektorsystem $\left(\mathfrak{a}_{1}, \ldots, \mathfrak{a}_{n}\right)$ in $\boldsymbol{R}^{n}$, dessen Existenz durch den Hilfssatz 1 für jedes $n \geqq 1$ gesichert ist, einer beliebigen orthogo- 
nalen Transformation (in $\boldsymbol{R}^{\boldsymbol{n}}$ ) unterworfen werden kann, so ist das Vektorsystem $\left(\mathfrak{c}_{1}, \ldots, \mathfrak{c}_{n+1}\right)$ nicht eindeutig bestimmt. Wir gehen darauf nicht näher ein.

3. Der Operator $S$ von H. A. Schwarz. Es sei $O$ eine fest gegebene offene, nicht leere Teilmenge von $\boldsymbol{R}^{\boldsymbol{n}}$. Wir ordnen jedem Punkt $x$ von $O$ ein (lokales) Vektorsystem

$$
H_{x}=\left(\mathfrak{h}_{1}, \ldots, \mathfrak{h}_{n+1}\right)
$$

zu mit den Eigenschaften (2.1), insbesondere mit der Eigenschaft

$$
\left\langle\mathfrak{h}_{i}, \mathfrak{h}_{k}\right\rangle=\left\{\begin{array}{rc}
1 & (i=k) \\
-\frac{1}{n} & (i \neq k)
\end{array},\right.
$$

und schreiben $h H_{x}$ für das Vektorsystem $\left(h \mathfrak{h}_{1}, \ldots, h \mathfrak{h}_{n+1}\right)$. Die Gesamtheit sämtlicher Vektorsysteme $H_{x}$ mit den Eigenschaften (3.2) wird im folgenden durch $\mathfrak{H}_{x}$ bezeichnet. Falls eine Abkürzung erforderlich ist, wird anstelle $H_{\boldsymbol{x}}$ und $\mathfrak{S}_{\boldsymbol{x}}$ die Schreibweise $H$ bzw. $\mathfrak{S}$ verwendet.

Definition 1. Im folgenden soll bedeuten:

1. $S(x, r)$ die offene (n-dimensionale) Kugel um den Punkt $x$ vom Radius $r$.

2. $\Omega_{x}^{r}$ den Rand von $S(x, r)$.

3. $\bar{S}(x, r)$ die abgeschlossene Kugel um $x$ vom Radius $r$.

4. Für $x \in O, S_{x}$ die grösste offene Kugel um $x$, die in $O$ liegt.

Der Radius von $S_{x}$ ist stets positiv oder $+\infty$.

Definition 2. Bei gegebenem $x \in O$ soll

$$
A_{h H} f(x)=\frac{1}{n+1} \sum_{k=1}^{n+1} f\left(x+h \mathfrak{h}_{k}\right)
$$

gesetzt werden. Dabei wird vorausgesetzt, dass $h$ so gewählt wird, dass die Punkte $x+h \mathfrak{h}_{k}$ in $S_{x}$ liegen.

Definition 3. Für jedes $x \in O$ und jedes $H_{x} \in \mathfrak{S}_{x}$ wird der Operator $S_{h H} f(x)$ durch die Gleichung

$$
S_{h H} f(x)=\frac{2 n}{h^{2}}\left[A_{h H} f(x)-f(x)\right]
$$

definiert.

Für $n=1$ und somit $O$ gleich einer Vereinigung von offenen Intervallen von $\boldsymbol{R}^{\mathbf{1}}$ stimmt dieser Operator mit dem klassischen Ausdruck von H. A. Schwarz ${ }^{3}$ )

3) Man vgl. etwa [5], S. 341 f. 


$$
\frac{f(x+h)+f(x-h)-2 f(x)}{h^{2}}
$$

überein. Der Operator $S_{h H} f(x)$ wird hier als der Schwarzsche Operator bezeichnet werden.

Innerhalb $\mathfrak{H}_{x}$ können folgende Operationen definiert werden:

1. Die Operation.

$$
\underline{S} f(x)=\lim _{h \rightarrow 0} S_{h H} f(x) .
$$

Dabei wird die Bildung, sofern nichts gegenteiliges vermerkt wird, unter Heranziehung sämtlicher $H_{x}$ durchgeführt.

2. Die Operation

$$
\bar{S} f(x)=\varlimsup_{h \rightarrow 0} S_{h H} f(x) .
$$

Ist $\quad \underline{S} f(x)=\bar{S} f(x)$, so wird dafür $S f(x)$ geschrieben. Diese Schreibweise auf der linken Seite einer Gleichung soll im folgenden stets zum Ausdruck bringen, dass der Grenzwert existiert und gleich dem Ausdruck auf der rechten Seite ist.

4. Der Laplacesche und der Blaschke-Privaloffsche Operator. Während die Definition des Operators $S_{h H} f$ lediglich an die Bedingung $f \in C[O]$ geknüpft ist, erfordert die Definition des Laplaceschen Operators die Zugehörigkeit von $f$ zu der Menge $C^{2}[O]$ der zweimal stetig differenzierbaren Funktionen in $O$.

Definition 4. Der (Vektor-)Operator

$$
\nabla=\left(\frac{\partial}{\partial x_{1}}, \ldots, \frac{\partial}{\partial x_{n}}\right)=\left(\partial_{x_{1}}, \ldots, \partial_{x_{n}}\right)
$$

soll der Hamilton- bzw. der Nabla-Operator heissen. Setzt man $\Delta=\nabla^{2}=$ $\langle\nabla, \nabla\rangle$, so soll der Operator

$$
\Delta=\frac{\partial^{2}}{\partial x_{1}^{2}}+\ldots+\frac{\partial^{2}}{\partial x_{n}^{2}}=\partial_{x_{1}}^{2}+\ldots+\partial_{x_{n}}^{2}
$$

der Laplacesche Operator heissen.

Im folgenden wird der Operator $\Delta$ auf Funktionen von $C^{2}[O]$ angewandt.

Defintion 5. Es bezeichne $C_{0}[O]$ die Gesamtheit sämtlicher auf $O$ definierten Funktionen $f$ mit den Eigenschaften: 1$)-\infty \leqq f<+\infty$, 2) $f$ ist halbstetig nach oben. Es sei $f \in \underset{\wedge}{C}[O], x \in O$ und $S(x, h) \subset S_{x}$. Dann wird gesetzt 


$$
\begin{aligned}
& P_{h} f(x)=\frac{1}{h^{2}} \int_{\Omega_{0}^{1}}[f(x+h y)-f(x)] d \omega(y), \\
& \underline{P} f(x)=\lim _{h \rightarrow 0} P_{h} f(x)
\end{aligned}
$$

und

$$
\bar{P} f(x)=\varlimsup_{h \rightarrow 0} P_{h} f(x) .
$$

Dabei bedeutet $\Omega_{0}^{1}$ den Rand von $S(0,1), y$ einen Punkt von $\Omega_{0}^{1}$ und $d \omega(y)$ das durch die Gleichung

$$
\int_{\Omega_{0}^{1}} d \omega(y)=1
$$

normierte Masselement von $\Omega_{0}^{1}$. Das Integral rechts ist im allgemeinen im Lebesgueschen Sinne zu nehmen $\left.{ }^{4}\right)$.

Die Operatoren $P_{h} f(x), \underline{P} f(x)$ und $\bar{P} f(x) \quad$ werden als BlaschkePrivaloffsche Operatoren bezeichnet. Ist $\underline{P} f(x)=\bar{P} f(x)$, so wird dafür kurz $P f(x)$ geschrieben. Legt man zunächst den Raum $C^{2}[O]$ zugrunde, so erhebt sich die Frage nach der Äquivalenz der Operatoren $S$, $\Delta$ und $P$. Die Äquivalenz von $P f$ und $\Delta f$ ist bekannt und wird in der Brelotschen Monographie [1] in grosser Allgemeinheit behandelt. In der nächsten Nummer soll zunächst die Äquivalenz von $S$ und $A$ gezeigt werden.

5. Definition der harmonischen Funktion. Zusammenhang mit dem Operator $S$. Wir bringen zunächst die Definition der harmonischen Funktion:

Definition 6. Die in $O$ definierte reelluertige Funktion $f$ heisst dort harmonisch, wenn sie dort die Eigenschaften besitzt:

1. Sie genügt für hinreichend kleine $x^{\prime}-x$ der Gleichung

$$
f\left(x^{\prime}\right)=e^{<x^{\prime}-x, \nabla>} f(x)
$$

(lokale Entwickelbarkeit in eine konvergente Taylor-Reihe).

2. Sie genügt in jedem Punkt $x$ von $O$ der (Laplaceschen) Differentialgleichung

$$
\Delta f(x)=\sum_{k=1}^{n} \partial_{x_{k} x_{k}}^{2} f(x)=\sum_{k=1}^{n} f_{x_{k} x_{k}}(x)=0 .
$$

4) Zur allgemeinen Orientierung vgl. man [1] und [6]. 
Der Raum der auf $O$ definierten harmonischen Funktionen wird im folgenden durch $H[O]$ bezeichnet werden. Es gehört zu den klassischen Ergebnissen der Theorie der harmonischen Funktionen, dass der Raum $H[O]$ mit folgendem Funktionenraum übereinstimmt:

$$
L[O]=\left\{f \mid f \in C^{2}[O], \Delta f=0\right\} .
$$

Unser Ziel ist der Beweis des Satzes:

Satz 1. (Äquivalenzsatz.) Man definiere den Funktionenraum $S[O]$ durch die Gleichung

$$
S[O]=\{f \mid f \in C[O], \underline{S} f \leqq 0 \text { und } \bar{S} f \geqq 0\} .
$$

Dann ist $\quad S[O]=H[O]$.

Mit anderen Worten: Jede stetige Funktion $f$ in $O$, die in jedem Punkt $x$ von $O$ den Ungleichungen $\underline{S} f(x) \leqq 0$ und $\bar{S} f(x) \geqq 0$ genügt, ist harmonisch in $O$.

Wir beweisen zunächts den Satz:

Satz 2. Für jedes $f \in C^{2}[O]$ gilt die Gleichung

$$
S f(x)=\Delta f(x) \text {. }
$$

Beweis. Es sei $H=\left(\mathfrak{h}_{1}, \ldots, \mathfrak{h}_{n+1}\right)$ ein System aus $\mathfrak{H}$ mit $\mathfrak{h}_{k}=\underset{k}{\left(h_{1}, \ldots, h_{k}\right)} \quad(k=1, \ldots, n+1)$. Dann ist

$$
\begin{aligned}
& \sum_{k=1}^{n+1}\left\{f\left(x+h \mathfrak{h}_{k}\right)-f(x)\right\} \\
& =h \sum_{k=1}^{n+1}\left\langle\mathfrak{h}_{k}, \nabla\right\rangle f(x)+\frac{h^{2}}{2} \sum_{k=1}^{n+1}\left\langle\mathfrak{h}_{k}, \nabla\right\rangle^{2} f(x)+o\left(h^{2}\right) .
\end{aligned}
$$

Nun ist

$$
\sum_{k=1}^{n+1}\left\langle\mathfrak{h}_{k}, \nabla\right\rangle f(x)=\sum_{k=1}^{n+1}\left[\sum_{\mu=1}^{n} h_{k} f_{x_{\mu}}\right]=\sum_{\mu=1}^{n}\left[\sum_{k=1}^{n+1} h_{\mu}\right] f_{x_{\mu}}=0
$$

und

$$
\begin{aligned}
& \sum_{k=1}^{n+1}\left\langle\mathfrak{h}_{k}, \nabla\right\rangle^{2} f(x)=\sum_{k=1}^{n+1}\left[\sum_{i=1}^{n} h_{k} \partial_{x_{i}}\right]^{2} f(x) \\
& =\sum_{\lambda, \mu=1}^{n}\left\langle\mathfrak{h}_{\mu}^{\prime}, \mathfrak{h}_{\lambda}^{\prime}\right\rangle f_{x_{\mu} x_{\lambda}}=\frac{n+1}{n} \sum_{\lambda, \mu=1}^{n} \delta_{\lambda, \mu} f_{x_{i} x_{\mu}} .
\end{aligned}
$$

Somit ist

$$
S_{h H} f(x)=\Delta f(x)+o(1) .
$$

Das beweist den Satz 2 . 
$\mathrm{Zu}$ bemerken wäre hier, dass beim Grenzübergang $h \rightarrow 0$ das Vektorsystem $H=\left(\mathfrak{h}_{1}, \ldots, \mathfrak{h}_{n+1}\right)$ festgehalten werden kann. Im allgemeinen jedoch gilt (5.6) unabhängig von $H$.

6. Der Äquivalenzsatz. Es bedeutet für die nachfolgenden Entwicklungen offenbar keine Einschränkung der Allgemeinheit, wenn man $O$ zusammenhängend, d. h. ein Gebiet nimmt.

Da aus dem Satz 2 die Ungleichung $L[O] \subset S[O]$ und somit $H[O] \subset S[O]$ folgt, so genügt es zum Beweis des Satzes 1, lediglich die Ungleichung $S[O] \subset H[O]$ zu beweisen.

Es sei $x_{0} \in O, \quad \bar{S}\left(x_{0}, r\right) \subset S_{x_{0}}$ und $v$ die harmonische Funktion in $S\left(x_{0}, r\right)$ mit den (stetigen) Randwerten $f(x)$ auf dem Rande $\Omega_{x_{0}}^{r}$ von $S\left(x_{0}, r\right)$. Man setze bei gegebenem $\varepsilon>0$

$$
g_{\varepsilon}(x)=f(x)-v(x)+\varepsilon\left\|x-x_{0}\right\|^{2}=g(x)+\varepsilon\left\|x-x_{0}\right\|^{2},
$$

und nehme an, die Ungleichung

$$
\bar{S} f(x) \geqq 0
$$

gelte in jedem Punkt von $O$. Dann wird wegen (5.5)

$$
\bar{S} g_{\varepsilon}(x) \geqq(n+1) \varepsilon \text {. }
$$

Es sei nun

$$
M=\sup \left\{g_{\varepsilon}(x) \mid x \in S\left(x_{0}, r\right)\right\},
$$

und für ein $x_{1} \in S\left(x_{0}, r\right), g_{\varepsilon}\left(x_{1}\right)=M$.

Dann würde wegen

$$
S_{h H} g_{\varepsilon}\left(x_{1}\right)=\frac{2 n}{h^{2}}\left[A_{h H} g_{\varepsilon}\left(x_{1}\right)-g_{\varepsilon}\left(x_{1}\right)\right] \leqq \frac{2 n}{h^{2}}[M-M]=0
$$

die Ungleichung $\bar{S} g_{\varepsilon}\left(x_{1}\right) \leqq 0$ folgen, was der Ungleichung (6.2) widerspricht. Somit gilt

d. h. (wegen $v(x)-f(x) \rightarrow 0$ für $\quad\left\|x-x_{0}\right\| \rightarrow r$ )

$$
\sup \left\{g_{\varepsilon}(x) \mid x \in S\left(x_{0}, r\right)\right\} \leqq \varlimsup_{\left|: x-x_{0}\right| \rightarrow r} g_{\varepsilon}(x),
$$

$$
f(x)-v(x)+\varepsilon\left\|x-x_{0}\right\|^{2} \leqq \varepsilon r^{2} .
$$

Das liefert durch Grenzübergang die Ungleichung

$$
f(x) \leqq v(x) \quad\left(x \in S\left(x_{0}, r\right)\right) .
$$

Durch Anwendung des gleichen Verfahrens auf die Funktion

$$
v(x)-f(x)+\varepsilon\left\|x-x_{0}\right\|^{2},
$$


erhält man entsprechend

$$
v(x) \leqq f(x) \quad\left(x \in S\left(x_{0}, r\right)\right),
$$

und mithin in Verbindung mit (6.3)

$$
f(x)=v(x) \quad\left(x \in S\left(x_{0}, r\right)\right) .
$$

Das beweist die Ungleichung $S[O] \subset H[O]$.

Somit ist folgender Satz bewiesen worden:

Satz 3. Jede in $O$ reellwertige, stetige Funktion $f$ mit den Eigenschaften $\bar{S} f \geqq 0$ und $\underline{S} f \leqq 0$ ist harmonisch in $O$.

Nachfolgender Satz kann als Verallgemeinerung des klassischen Gauss'schen Mittelwertsatzes angesehen werden:

Satz 4. Man ordne jedem Punkt $x$ von $O$ ein festes, jedesmal von $x$ abhängiges Vektorsystem $H_{x}=\left(\mathfrak{h}_{1}, \ldots, \mathfrak{h}_{n+1}\right)$ zu und nehme an, dass die Funktion $f \in C[O]$ die Eigenschaft

$$
\lim _{h \rightarrow 0} S_{h H} f(x)=0
$$

besitzt. Dann ist $f$ harmonisch in $O$.

Da die Bedingung (6.5) schwächer als die Gauss'sche Bedingung

$$
\int_{\Omega_{0}^{1}} f(x+h y) d \omega(y)=f(x)
$$

ist, folgt daraus, dass (6.6) erst dann aus (6.5) folgt, wenn letztere für alle Systeme $H_{x}$ von $\mathfrak{S}_{x}$ gilt.

7. Die subharmonische Klasse. Verallgemeinerung des Kriteriums von Blaschke-Privaloff. Zunächst sei die Definition der subharmonischen Funktion in $\boldsymbol{R}^{n}$ vorangeschickt:

Definition 7. Eine auf $O$ definierte numerische Funktion $f$ soll subharmonisch heissen, wenn sie folgenden Bedingungen genügt:

(1) $f(x) \in \wedge \boldsymbol{R}=[-\infty,+\infty), \quad f(x) \neq-\infty$.

(2) $f$ ist halbstetig nach oben.

(3) Es sei $x$ ein beliebiger Punkt von $O$ und $\bar{S}(x, r) \subset S_{x}$. Ferner sei $h$ eine in $\bar{S}(x, r)$ stetige und in $S(x, r)$ harmonische Funktion mit $f \leqq h$ auf dem Rand $\Omega_{x}^{r}$ von $S(x, r)$. Dann gilt die Ungleichung $f \leqq h$ in $S(x, r)$.

Die Gesamtheit sämtlicher auf $O$ subharmonischen Funktionen wird durch $\underset{\wedge}{H}[O]$ bezeichnet. 
Der Satz von Blaschke-Privaloff (Brelot [1]) kann nun folgender- . massen formuliert werden:

Satz 5. (Blaschke-Privaloff.) Es sei $f \in C[O]$. Genügt dann $f$ in jedem Punkt $x$ der Menge

$$
O_{0}:=\{x \mid x \in O,-\infty<f(x)<+\infty\}
$$

der Bedingung

$$
\bar{P} f(x) \geqq 0,
$$

so ist $f$ subharmonisch in $O$.

Wegen $f \neq-\infty$ ist die Menge $O_{0}$ nicht leer.

Das Kriterium von Blaschke und Privaloff hat ein Analogon im Raum $C[O]$ sämtlicher auf $O$ definierten, nach oben halbstetigen (numerischen) Funktionen $f \geqq-\infty$.

Satz 6. Es sei $f \in \underset{\wedge}{C}[O]$. Genügt dann $f$ der Ungleichung

$$
\bar{S} f(x) \geqq 0
$$

in jedem Punkt $x$ von $O_{0}$, so ist $f$ subharmonisch in $O$.

Beweis. Es genügt offenbar zu beweisen, dass die Bedingung (7.3) die Bedingung (3) der Definition 7 impliziert. Es bezeichne $x_{0}$ einen Punkt von $O_{0}$ und $S\left(x_{0}, r\right) \subset S_{x_{0}}$ eine Kugel um $x_{0}$ vom Radius $r>0$. Man konstruiere eine in $S\left(x_{0}, r\right)$ harmonische Funktion $h$ mit stetigen Randwerten $\geqq f$ auf $\Omega_{x_{0}}^{r}$. Wir setzen, ähnlich wie vorhin, bei gegebenem $\varepsilon>0$

$$
g_{\varepsilon}(x)=f(x)-h(x)+\varepsilon\left\|x-x_{0}\right\|^{2},
$$

und bilden $\bar{S} g_{\varepsilon}(x)$ in jedem Punkt von $O_{0}$ in $S$. Da (7.3) gilt, so existieren für beliebig kleine $h$ Systeme $h H$ mit endlichem $S_{h H} f(x)$. Nun folgt aus

$$
S_{h H} g_{\varepsilon}(x)=S_{h H} f(x)-S_{h H} h(x)+2 n \varepsilon
$$

und somit wieder (wegen $S h(x)=0$ )

$$
\bar{S} g_{\varepsilon}(x)=\bar{S} f(x)+2 n \varepsilon \geqq(n+1) \varepsilon .
$$

Daraus folgt, dass $g_{\varepsilon}$ in keinem Punkt von $O_{0}$ ein Maximum haben kann. Somit gibt es mindestens eine Cauchy-Folge $\left[y_{k}\right]_{1}^{\infty}$ von $O_{0}$ in $S\left(x_{0}, r\right)$ mit den Eigenschaften:

1.

$$
\lim _{k \rightarrow+\infty} y_{k}=\widehat{x} \in \bar{S}\left(x_{0}, r\right)-O_{0} .
$$

2.

$$
\lim _{k \rightarrow+\infty} g_{\varepsilon}\left(y_{k}\right)=M=\sup \left\{g_{\varepsilon}(x) \mid x \in S\left(x_{0}, r\right)\right\} \text {. }
$$


Nun ist $f$ halbstetig nach oben und somit auch $g_{\varepsilon}$. Das hat zur Folge, dass

$$
g_{\varepsilon}(x) \leqq \varlimsup_{\left\|x-x_{0}\right\| \rightarrow r} g_{\varepsilon}(x) \quad\left(x \in S\left(x_{0}, r\right)\right)
$$

gilt und wegen

wird

$$
\varlimsup_{\left\|x-x_{0}\right\| \rightarrow r}(f(x)-h(x)) \leqq 0
$$

$$
g_{\varepsilon}(x)=f(x)-h(x)+\varepsilon\left\|x-x_{0}\right\|^{2} \leqq \varepsilon r^{2} \quad\left(x \in S\left(x_{0}, r\right)\right) .
$$

Der Grenzübergang $\varepsilon \rightarrow 0$ liefert den Beweis $f \leqq h$ in $S\left(x_{0}, r\right)$ und somit die Erfüllbarkeit der Bedingung (3) der Definition 7.

Der Vorteil des Operators $S$ gegenüber dem Operator $P$ von Blaschke-Privaloff liegt an erster Stelle darin, dass er ausser dem Grenzübergang $h \rightarrow 0$ keine weiteren Mittelungsprozesse, wie etwa den Differentiations- bzw. Integrationsprozess benötigt.

Aus der Tatsache, dass man durch Integration von $S_{h H_{x}} f(x)$ über sämtliche $H_{x}$ den Operator $P_{h} f(x)$ erhält, folgt, dass die Forderung $\bar{S} f(x) \geqq 0 \quad$ in gewisser Hinsicht schwächer als die Bedingung $\bar{P} f(x) \geqq 0$ ist. Darauf soll jedoch hier nicht näher eingegangen werden.

8. Der Fall eines Riemannschen Raumes. Die vorherigen Entwicklungen lassen sich ohne Schwierigkeit auf Riemannsche Räume übertragen. Wir erinnern zunächst kurz an folgende Begriffsbildungen:

Unter einer $n$-dimensionalen Mannigfaltigkeit wird allgemein ein topologischer separierbarer Raum $X$ verstanden, dessen Punkte mindestens eine Umgebung besitzen, die zu der offenen $n$-dimensionalen euklidischen Einheitskugel homöomorph ist $\left.{ }^{5}\right)$. Es wird vorausgesetzt, dass $X$ eine abzählbare Überdeckung $\left\{U_{i}\right\}_{1}^{\infty}$ besitzt derart, dass jede offene Teilmenge von $X$ sich als Vereinigung von Mengen $U_{i}$ darstellen lässt. Da mit $U \in\left\{U_{i}\right\}_{1}^{\infty}$ zugleich die Abbildung $x \rightarrow\left(x^{1}, \ldots, x^{n}\right)$ in die Einheitskugel $S(0,1)$ (ausführlicher: $\left(x^{1}(x), \ldots, x^{n}(x)\right)$ ) gegeben wird, so lässt sich die Gesamtheit der Funktionen $f$ auf $X$ durch die Gleichung

$$
f(x)=f\left(x^{1}, \ldots, x^{n}\right)
$$

definieren. Die Funktion $f$ ist definiert auf $X$, wenn sie in der Umgebung jedes Punktes von $x$ durch die Vorschrift (8.1) definiert ist. Ist $r \geqq 0$ ganz, so bedeutet die Schreibweise $f \in C^{r}$, dass $f$ (ausgedrückt durch die lokalen Koordinaten $x^{1}, \ldots, x^{n}$ stetige Ableitungen

${ }^{5}$ ) Zur allgemeinen Orientierung vgl. man [9]. 
von der Ordnung $k \leqq r$ hat. Insbesondere bedeutet $C$ die Gesamtheit sämtlicher stetigen Funktionen auf $X$.

Wird $U$ durch zwei Koordinatensysteme $\left(x^{1}, \ldots, x^{n}\right)$ und $\left(x^{\prime 1}, \ldots, x^{\prime n}\right)$ (kurz: $\left(x^{k}\right)$ und $\left(x^{\prime k}\right)$ ) gegeben, so werden die Transformationssysteme

$$
\begin{aligned}
x^{\prime k} & =x^{\prime k}\left(x^{1}, \ldots, x^{n}\right) \\
x^{k} & =x^{k}\left(x^{\prime}, \ldots, x^{\prime n}\right)
\end{aligned} \quad(k=1, \ldots, n)
$$

im allgemeinen durch Funktionen $x^{k}, x^{\prime k}$ hergestellt, die in $C^{r}(r \geqq 3)$ liegen. Bei jeder Transformation von der Art (8.2) (lokale Koordinatentransformation) wird angenommen, dass beide Determinanten

$$
\left|\frac{\partial x^{\prime k}}{\partial x^{l}}\right|,\left|\frac{\partial x^{k}}{\partial x^{\prime l}}\right|
$$

von Null verschieden sind.

Definition 8. Ein Riemannscher Raum wird auf einer n-dimensionalen Mannigfaltigkeit $X$ durch die Vorgabe einer positiv definiten quadratischen Differentialform

$$
d s^{2}=g_{i k} d x^{i} d x^{k} \quad(i, k=1, \ldots, n)
$$

mit $\quad g_{k i}=g_{i k}, \quad g_{i k}=g_{i k}\left(x^{1}, \ldots, x^{n}\right)=g_{i k}(x)$ gegeben $\left.{ }^{6}\right)$. Die Form (8.3) soll im Sinne der Gleichung

$$
d s^{2}=g_{i k} \frac{\partial x^{i}}{\partial x^{\prime \mu}} \frac{\partial x^{k}}{\partial x^{\prime \nu}} d x^{\prime \mu} d x^{\prime \nu}=g_{\mu \nu}^{\prime} d x^{\prime \mu} d x^{\prime \nu}
$$

invariant sein.

Wir schreiben im folgenden $M^{n}$ für den dadurch definierten Riemannschen Raum und nehmen an, dass die $g_{i k}$ mindestens einmal nach den jeweiligen lokalen Koordinaten stetig differenzierbar sind.

Definition 9. Es wird geschrieben: ${ }^{7}$ )

(1) $g$ für die (positive) Determinante der $g_{i k}$.

(2) $g^{i k}$ für die Ausdrücke $A_{i k} / g$, wobei $A_{i k}$ das algebraische Komplement von $\left(g_{i k}\right)$ in bezugauf $g_{i k}$ ist. Bekanntlich gelten die Gleichungen

$$
g^{i_{\mu}} g_{\mu k}=\delta_{k}^{i}=\delta_{i k}=\left\{\begin{array}{ll}
1 & (i=k) \\
0 & (i \neq k)
\end{array} .\right.
$$

Es gilt stets $g^{k i}=g^{i k}$.

${ }^{6}$ ) Als Nachschlagswerk für den klassischen Tensorkalkül kann man [2] benutzen Sowohl in (8.3) wie auch später wurde im Einklang mit der bekannten Vorschrift des Tensorkalküls bei mehrfach auftretenden Indizes das Summationszeichen weggelassen. In solchen Fällen ist dann stets über den betreffenden (stummen) Index von 1 bis $n$ zu summieren.

${ }^{7}$ Man vgl. [2], S. $27 \mathrm{ff}$. 
(3) $\Gamma_{k l}^{i}$ für die Ausdrücke (Gamma-Symbole) von Christoffel

$$
\frac{1}{2} g^{i_{\mu}}\left[\begin{array}{c}
k l \\
\mu
\end{array}\right]=\left\{\begin{array}{c}
k l \\
i
\end{array}\right\}=\frac{1}{2} g^{i \mu}\left(\frac{\partial g_{k \mu}}{\partial x^{l}}+\frac{\partial g_{l_{\mu}}}{\partial x^{k}}-\frac{\partial g_{k l}}{\partial x^{\mu}}\right) .
$$

(4) $\Delta$ für den allgemeinen Laplaceschen Operator

$$
\frac{1}{\sqrt{g}} \frac{\partial}{\partial x^{k}}\left(\sqrt{g} g^{k l} \frac{\partial}{\partial x^{l}}\right) \text {. }
$$

Dieser geht für $g_{i k}=\delta_{i k}$ in den klassischen Operator

$$
\frac{\partial^{2}}{\partial x^{1} \partial x^{1}}+\ldots+\frac{\partial^{2}}{\partial x^{n} \partial x^{n}}=\partial_{x_{1}}^{2}+\ldots+\partial_{x_{n}}^{2}
$$

über.

Neben diesen Definitionen wird im folgenden noch das Differentialgleichungssystem

$$
\frac{d^{2} x^{i}}{d s^{2}}+\Gamma_{k l}^{i} \frac{d x^{k}}{d s} \frac{d x^{l}}{d s}=0 \quad(i, k=1, \ldots, n)
$$

mit der Bedingung

$$
g_{i k} \frac{d x^{i}}{d s} \frac{d x^{k}}{d s}=1
$$

benötigt $\left.{ }^{8}\right)$.

9. Vorbereitende Tatsachen. Es bezeichne $O$ eine nicht leere offene Teilmenge von $M^{n}, \quad f$ eine reellwertige, zweimal stetig differenzierbare Funktion in bezug auf die jeweiligen lokalen Koordinaten (also $\left.f \in C^{2}[O]\right), \quad x$ einen festen sonst beliebigen Punkt von $O$ und $\gamma$ einen geodätischen Kurvenbogen durch $x$ mit dem durch die Gleichung $g_{i k} \xi^{i} \xi^{k}=1$ normierten Tangentenvektor $\xi=\left(\xi^{1}, \ldots, \xi^{n}\right)$ in $x$. Die Bogenlänge $s$ von $\gamma$ soll von $x$ aus gemessen werden. Dann kann man die Koordinaten der Punkte $y=\left(y^{1}, \ldots, y^{n}\right)$ von $\gamma$ in der Umgebung von $x$ in der Form ${ }^{9}$ )

$$
x^{i}+\psi_{i}\left(s \xi^{1}, \ldots, s \xi^{n}\right)
$$

darstellen, wobei die $\psi_{i}$ dem System (8.8) genügen, und die Bedingungen $\psi_{i}^{\prime}(0, \ldots, 0)=0, \quad\left(d \psi_{i} / d s\right)_{s=0}=\psi_{i}^{\prime}(0, \ldots, 0)=\xi^{i} \quad(i=1, \ldots, n)$ erfüllen.

8) Man vgl. [3], S. $40 \mathrm{ff}$.

9) Eine ausgezeichnete Darstellung der Riemannschen Zentralkoordinaten findet der Leser in den Weylschen Kommentaren in [4]. 
Bekanntlich definieren die Gleichungen $x^{i}+\psi_{i}$ die (infinitesimale) Parallelverschiebung von $\xi$ längs $\gamma$.

Eine leichte Rechnung bestätigt nun die Gleichungen

$$
\left(\frac{d f}{d s}\right)_{s=0}=f_{x^{k}} \xi^{k}
$$

und (mit Rücksicht auf das System (8.8))

$$
\left(\frac{d^{2} f}{d s^{2}}\right)_{s=0}=\left(f_{x^{k} x^{l}}-\Gamma_{k l}^{\mu} f_{x^{u}}\right) \xi^{k} \xi^{l} .
$$

Führt man in (9.2) die kovarianten Komponenten $\xi_{i}$ von $\xi$ durch die Gleichungen

$$
\xi_{i}=g_{i \mu} \xi^{\mu}
$$

ein, so lässt sich (9.2) in die Form

$$
\left(\frac{d^{2} f}{d s^{2}}\right)_{s=0}=g^{k v}\left(f_{x^{k} x^{l}}-\Gamma_{k l}^{\mu} f_{x^{u}}\right) \xi_{v} \xi^{l}
$$

bringen.

Diese Gleichung, die praktisch die zweite Richtungsderivierte von $f$ in $x$ auf $\gamma$ gibt, bildet die Grundlage der weiteren Untersuchung.

10. Der Operator $S$. Die Übertragung des Operators $S_{h H}$ auf Riemannsche Mannigfaltigkeiten erfordert lediglich den Begriff der Parallelverschiebung des Tangentenvektors auf einer geodätischen Linie durch den Punkt $x$. Die Vektorsysteme $H=\left(\mathfrak{h}_{1}, \ldots, \mathfrak{h}_{n+1}\right)$ liegen jetzt im Tangentialraum von $M^{n}$ in $x$ und bestimmen durch ihre Vektoren $\mathfrak{h}_{1}, \ldots, \mathfrak{h}_{n+1}$ (die hier die Rolle der $\xi^{k}$ übernehmen), jedesmal die $n+1 \quad n$-Tupel $\quad \underset{k}{\psi}=\left(\psi_{1}, \ldots, \psi_{n}\right) \quad(k=1, \ldots, n+1), \quad$ die jedesmal von $s$ und der Anfangsrichtung $\mathfrak{h}_{k}=\underset{k}{\left(h_{1}, \ldots, h_{n}\right)}$ abhängen.

Es sei jetzt $f$ reellwertig auf einer offenen Punktmenge $O$ von $M^{n}$. Wir setzen im Anschluss an die Entwicklungen von 3

$$
A_{s H} f(x)=\frac{1}{n+1} \sum_{k=1}^{n+1} f(x+\underset{k}{\psi})
$$

und definieren den Operator $S_{s H}$ auf $O$ durch die Gleichung

$$
S_{s H} f(x)=\frac{2 n}{s^{2}}\left\{A_{s H} f(x)-f(x)\right\} .
$$


Hat man die Definition von $s H$ (bei gegebenem $H_{x}$ ), so lassen sich $\underline{S} f(x), \quad \bar{S} f(x)$ und $S f(x)$ durch die Gleichungen

$$
\begin{aligned}
& \underline{S} f(x)=\lim _{s \rightarrow 0} S_{s H} f(x), \\
& \bar{S} f(x)=\varlimsup_{s \rightarrow 0} S_{s H} f(x)
\end{aligned}
$$

und

$$
S f(x)=\lim _{s \rightarrow 0} S_{s H} f(x)
$$

definieren. Letztere Gleichung setzt voraus, dass der Grenzwert rechts existiert, d. h. dass $\underline{S} f(x)=\bar{S} f(x)$ gilt.

Definition 10. Die in $O \subset M^{n}$ definierte reellwertige Funktion $f$ wird dort als harmonisch bezeichnet, wenn sie in jedem Punkt $x$ von $O$ die Eigenschaften hat:

1. Die Ableitungen $f_{x^{k} x^{l}}$ (genommen in bezug auf die lokalen Koordinaten) existieren in jedem Punkt $x$ von $O$ und sind dort stetig.

2. Es gilt

$$
\Delta f(x)=\frac{1}{\sqrt{g}} \frac{\partial}{\partial x^{k}}\left(\sqrt{g} g^{k l} \frac{\partial}{\partial x^{l}} f(x)\right)=0
$$

in jedem Punkt von $O$.

Dass in der Definition 10 die lokale Darstellbarkeit von $f$ im Sinne der Gleichung (5.1) nicht zugrundegelegt wird, liegt darin, dass die $g_{i k}$ im allgemeinen nicht analytische Funktionen der lokalen Koordinaten sind.

Man kann hier jedoch ohne Schwierigkeit das Analogon des Satzes 2 beweisen:

Satz 7. Für jedes $f \in C^{2}[O]$ gilt die Gleichung

$$
S f(x)=\Delta f(x) .
$$

Dabei wird $\triangle$ durch (8.7) gegeben.

Beweis. Es sei $f \in C^{2}[O]$. Dann ist

$$
J=(n+1) \lim _{s \rightarrow 0} S_{s H} f(x)=n \sum_{k=1}^{n+1}\left[g^{r v}\left(f_{x^{r} x^{l}}-\Gamma_{r l}^{\mu} f_{x^{\mu}}{ }_{\substack{k \\ k}}^{h_{v}} h_{k}\right],\right.
$$

und somit wegen

$$
\begin{gathered}
\sum_{k=1}^{n+1} h_{v} h_{k} h_{l}=\left\langle\mathfrak{h}_{v}^{\prime}, \mathfrak{h}_{l}^{\prime}\right\rangle=\left\{\begin{array}{cc}
\frac{n+1}{n} & (v=l) \\
0 & (v \neq l)
\end{array} \quad(v, l=1, \ldots, n),\right. \\
S f(x)=g^{r l}\left(f_{x^{r} x^{l}}-\Gamma_{r l}^{\mu} f_{x^{u}}\right) .
\end{gathered}
$$


Die Heranziehung der Identitäten ${ }^{10}$ )

$$
g^{r l} \Gamma_{r l}^{\mu}+\frac{1}{\sqrt{ } g} \frac{\partial}{\partial x^{l}}\left(\sqrt{g} g^{u l}\right)=0
$$

liefert nun durch Einsetzen

$$
\begin{aligned}
S f(x) & =g^{r l} f_{x^{r} x^{l}}+\frac{1}{\sqrt{g}} \frac{\partial}{\partial x^{\mu}}\left(\sqrt{g} g^{u l}\right) f_{x^{l}} \\
& =\frac{1}{\sqrt{g}} \frac{\partial}{\partial x^{k}}\left(\sqrt{g} g^{k l} \frac{\partial f}{\partial x^{l}}\right)=\Delta f(x) .
\end{aligned}
$$

Somit gilt für jedes $x \in O$

$$
\Delta f(x)=0 \Rightarrow S f(x)=0 \quad\left(f \in C^{2}[O]\right) .
$$

Der Beweis, dass aus $S f(x)=0$ in $O$ stets $\Delta f(x)=0$ folgt, kann genau so geführt werden, wie die entsprechende Behauptung für den Raum $\boldsymbol{R}^{n}$. Voraussetzung dafür ist hier die Existenz der Funktion $v$ in der Umgebung jedes Punktes $x$ von $O$, etwa innerhalb einer Kugel $\bar{S}(x, r)$ in $O$, welche auf $\Omega_{x}^{r}$ stetige Randwerte $\geqq f$ besitzt und der Potentialgleichung $\Delta v=0$ (wobei $\Delta$ durch (10.6) gegeben wird) genügt. Ist die Existenz von $v$ gesichert, so liefert die Wahl

$$
g_{\varepsilon}(y)=f(y)-v(y)+\varepsilon g_{i k}\left(y^{i}-x^{i}\right)\left(y^{k}-x^{k}\right)
$$

mit $g_{i k}=g_{i k}(x)$ das Analogon der Funktion $g_{\varepsilon}$ von 6. Die Durchführung des Beweises, unter Heranziehung der Operatoren $A_{s H}$ und $S_{s H}$ dieser Nummer, stösst auf keine Schwierigkeiten und wird dem Leser überlassen.

11. Nochmals der Blaschke-Privaloffsche Satz. Wird die Existenz der Funktion $v$ (lokal) vorausgesetzt, so können sowohl der BlaschkePrivaloffsche Satz als auch der Satz 5 auf die Räume $M^{n}$ übertragen werden. Zunächst ist die Bedeutung des Operators $P_{h} f(x)$ in dem Sinne zu modifizieren, dass hier anstelle des Integrals rechts in (4.3) der Ausdruck

$$
P_{s} f(x)=\frac{1}{s^{2}} \int_{\Omega_{x}^{1}}\{f(x+\psi)-f(x)\} d \omega(\xi)
$$

mit

$$
\psi=\left(\psi_{1}, \ldots, \psi_{n}\right)
$$

und

10) Man vgl. etwa [7], S. 119. 


$$
\psi_{k}=\psi_{k}\left(s \xi^{1}, \ldots, s \xi^{n}\right) \quad(k=1,2, \ldots, n)
$$

genommen wird. Dabei gilt wieder

$$
\psi_{k}(0, \ldots, 0)=0, \quad \psi_{k}^{\prime}(0, \ldots, 0)=\xi^{k},
$$

und

$$
g_{i k} \xi^{i} \xi^{k}=1 \quad\left(g_{i k}=g_{i k}(x)\right) .
$$

Ferner stellt $d \omega(\xi)$, ähnlich wie vorhin, das durch die Bedingung

$$
\int_{\Omega_{\boldsymbol{x}}^{1}} d \omega(\xi)=1
$$

normierte Masselement der euklidischen Kugel (11.2) in $\xi$ im Tangentialraum von $M^{n}$ in $x$ dar.

Mit Hilfe des Operators $P_{s} f(x)$ kann der Satz 5 dadurch verallgemeinert werden, dass man dort (unter der Annahme $f \in C[O]$ ) die Ungleichung (7.2) durch die Ungleichung

$$
\varlimsup_{s \rightarrow 0} P_{s} f(x) \geqq 0
$$

ersetzt.

Auf den Beweis dieses Satzes wird hier nicht eingegangen werden ${ }^{11}$ ). Nachfolgender Satz stellt das Analogon des Satzes 6 für den Riemannschen Raum $M^{n}$ dar:

Satz 8. Das Dirichletsche Problem sei (im Kleinen) lösbar in $M^{n}$. Es sei $f \in C[O]$ und

$$
\bar{S} f(x) \geqq 0
$$

in jedem Punkt $x$ der Menge

$$
O_{0}=\{x \mid x \in O,-\infty<f(x)<+\infty\} .
$$

Dann gilt $f \in H[O]$.

Der Beweis dieses Satzes verläuft nach dem Vorbild des Beweises des Satzes 6 und Heranziehung der Funktion

$$
g_{\varepsilon}(y)=f(y)-h(y)+\varepsilon g_{i k}(x)\left(y^{i}-x^{i}\right)\left(y^{k}-x^{k}\right)
$$

bei fest gegebenem $x$ in $O_{0}$ und $y \in \bar{S}(x, r) \subset S_{x}$. Hierbei bedeutet $h$, ähnlich wie in 7 , eine (in bezug auf den Operator (4) von 8) harmonische Funktion mit Randwerten $\geqq f$ auf $\Omega_{x}^{r}$. Bildet man wieder $\bar{S} g_{s}(x)$ (wobei $\bar{S}$ durch (10.4) gegeben wird), so erhält man wegen

11) Man vgl. [1], S. $18 \mathrm{f}$. 
$\bar{S} h(x)=0 \quad$ die Ungleichung $\bar{S} g_{\varepsilon}(x) \geqq(n+1) \varepsilon>0$. Der Rest des Beweises erfordert eine Wiederholung der Schlüsse in 7.

Die lokalen Eigenschaften von $S_{s \boldsymbol{H}}$, insbesondere die Definition der Operatoren $\underline{S}, \bar{S}$ und $S$ können einfacher begründet werden, wenn man die Riemannsche Form des Bogenelements von $M^{n}$ zugrundelegt ${ }^{12}$ ), welche den Vorteil besitzt, dass die lokalen (Ausgangs-)Koordinatensysteme orthogonal werden. Ich begnüge mich hier mit dieser Andeutung.

12. Anwendung auf die Theorie der elliptischen partiellen Differentialgleichungen. Das in den vorherigen Nummern entwickelte Kriterium für subharmonische Funktionen ohne Heranziehung des Integralbegriffs lässt sich zunächst ohne wesentliche Schwierigkeiten auf Lösungen von Differentialgleichungen von der Form

$$
\Delta u=g(x, u)
$$

in einem Gebiet $O$ von $\boldsymbol{R}^{\boldsymbol{n}}$ anwenden und ermöglicht den Nachweis der Existenz einer Lösung unter schwächeren Voraussetzungen als dies bisher der Fall war.

Satz 9. Es sei $g(x, y) \quad\left(x=\left(x_{1}, \ldots, x_{n}\right)\right)$ eine stetige Funktion von $(x, y)$ in $\boldsymbol{R}^{n} \times \boldsymbol{R}$ und $f$ eine auf $O \subset \boldsymbol{R}^{n}$ definierte reellwertige stetige Funktion von $x$ mit der Eigenschaft

$$
\begin{aligned}
& \underline{S} f(x) \leqq g(x, f(x)) \\
& \bar{S} f(x) \geqq g(x, f(x))
\end{aligned} \quad(x \in O) .
$$

Dann ist $f$ zweimal stetig differenzierbar und genügt der Differentialgleichung (12.1).

Es bezeichne in der Tat $\Phi$ eine (lokale) Lösung der Poissonschen Differentialgleichung

$$
\Delta \Phi(x)=g(x, f(x)) \quad\left(x \in S\left(x_{0}, r\right)\right) .
$$

Man setze $\Psi(x)=f(x)-\Phi(x)$ und beachte, dass (wegen $S \Phi(x)=\Delta(x)$ ?

$$
\begin{aligned}
& \frac{S}{T}(x)=S f(x)-\Delta \Phi(x)=\underline{S} f(x)-g(x \cdot f(x)), \\
& \bar{S} \Psi(x)=\overline{\bar{S}} f(x)-\Delta \Phi(x)=\bar{S} f(x)-g(x \cdot f(x))
\end{aligned}
$$

und somit $\underline{S} \Psi(x) \leqq 0$ und $\bar{S} \Psi(x) \geqq 0$ gilt. Daraus folgt, dass $\Psi$ harmonisch in $S\left(x_{0}, r\right)$ und somit in $O$ ist. Das hat zur Folge, dass $f$ zweimal stetig differenzierbar in $O$ ist und mithin gilt $\Delta f(x)=$ $S f(x)=g(x, f(x))$. Das beweist den Satz 9 .

Der Satz 9 kann dazu benutzt werden, nun die Existenz von differenzierbaren Lösungen der Schrödingerschen Differentialgleichung

12) Man vgl. [2], S. 234 ff. 


$$
\Delta \psi+\frac{8 \pi^{2} m}{h^{2}}(E-U) \psi=0
$$

wobei $m, h, E$ physikalische Konstanten sind und $U$ eine Ortsfunktion ist ${ }^{13}$ ), lediglich unter den Voraussetzungen

$$
\begin{aligned}
& S \psi+\frac{8 \pi^{2} m}{h^{2}}(E-U) \psi \leqq 0, \\
& \bar{S} \psi+\frac{8 \pi^{2} m}{h^{2}}(E-U) \psi \geqq 0
\end{aligned}
$$

mit einem stetigen $\psi, \mathrm{zu}$ beweisen. Der Beweis ist eine unmittelbare Folgerung des Satzes 9 und wird hier nicht wiederholt werden.

Da der Satz 9 auch dann gilt, wenn die Dimension $n$ gleich Eins genommen wird, so scheinen die Raum-Zeit-Vorgänge in der Natur weniger mit der zweimaligen Differenzierbarkeit der in Frage kommenden Funktionen zusammenzuhängen, als mit einer Oszillationseigenschaft des Operators $S$ (im euklidischen und Riemanmschen Raum), welche die Ungleichungen (12.2) und (12.5) und (für etwa die Zeitdimension)

$$
\begin{aligned}
& S y(t)+\lambda y(t) \leqq 0 \\
& \bar{S} y(t)+\lambda y(t) \geqq 0
\end{aligned} \quad(t \in R)
$$

mit einem Parameter $\lambda$ zur Folge hat. Dabei werden $\underline{S}, \bar{S}$ in (12.6) durch (3.5) gegeben $\left.{ }^{14}\right)$.

Folgende Bemerkung dürfte noch von Interesse sein: Bekanntlich ${ }^{15}$ ) führt das Entwicklungsproblem einer Funktion $f$ in $\boldsymbol{R}^{n}$ auf die Reihe $Y_{1}+Y_{2}+\ldots$ von Hyperkugelfunktionen, die wiederum auf $\Omega_{0}^{1}$ eingeschränkt, mit dem Problem der Entwicklung einer auf der Einheitskugel $\xi_{1}^{2}+\ldots+\xi_{n}^{2}=1$ definierten Funktion in eine Reihe $X_{1}+X_{2}+\ldots$ zusammenhängt, wobei die einzelnen $X_{k}$ den Gleichungen

$$
\text { d } X_{k}+k(k+n-2) X_{k}=0
$$

genügen, wobei $\triangle$ den Operator (4) von 8 für die Oberfläche der $n$-dimensionalen euklidischen Kugel (d. h. für $\Omega_{0}^{1}$ ) bedeutet. Man setze

$$
F(x)=\sum_{k=1}^{\infty} \frac{X_{k}(x)}{k(k+n-2)} \quad\left(x \in \Omega_{0}^{1}\right)
$$

13) Näheres über die Schrödingersche Differentialgleichung findet der Leser in jedem Buch über Theoretische Physik.

14) Dass ich hier dieses Beispiel gewählt habe, lag an meinem Wunsch zu demonstrieren, dass der Ablauf der Naturvorgänge auch ohne den Begriff der Differenzierbarkeit erfasst werden kann.

15) Zur allgemeinen Orientierung vgl. man etwa [7], S. $182 \mathrm{ff}$. 
und bilde $S F(x)$. Lassen sich hier Sätze beweisen, die die klassischen Sätze von Riemann über trigonometrische Reihen verallgemeinern? Eine entsprechende Frage lässt sich stellen, wenn man unter Zugrundelegung des Operators $S_{s H}$ von 10 das Analogon der Riemannschen Sätze für die Entwicklungen von Funktionen nach den Eigenfunktionen einer Sturm-Liouvilleschen Differentialgleichung beweisen kann. In diesem Falle würden die Eigenwerte $\lambda_{k}$ der in Frage kommenden Differentialgleichung die Rolle der $k(k+n-2)$ übernehmen. Das ist aber auch alles, was man augenblicklich dazu sagen kann.

Zum Schluss möchte ich Herrn H. Begehr in Berlin für Hilfe bei der Korrekturarbeit danken.

\author{
Freie Universität Berlin \\ Deutschland
}

\title{
Literatur
}

[1] Brelot, M.: Éléments de la théorie classique du potentiel. - [Zweite Auflage.] Les Cours de Sorbonne 3. Centre de Documentation Universitaire, Paris, 1961.

[2] Cartan, É.: Leçons sur la géométrie des espaces de Riemann. - [Zweite Auflage.] Cahiers Scientifiques II. Gauthier-Villars, Imprimeur-Éditeur, Paris, 1946.

[3] Courant, R., und D. HilberT: Methoden der mathematischen Physik I. - Die Grundlehren der mathematischen Wissenschaften XII. Verlag von Julius Springer, Berlin, 1924.

[4] Riemann, B.: Über die Hypothesen, welche der Geometrie zu Grunde liegen. Neu herausgegeben und erläutert von H. WEyL. Verlag von Julius Springer, Berlin, 1919.

[5] Schwarz, H. A.: Beweis eines für die Theorie der trigonometrischen Reihen in Betracht kommenden Hülfssatzes. - Gesammelte mathematische Abhandlungen II. Verlag von Julius Springer, Berlin, 1890, S. 341-343.

[6] Tsus, M.: Potential theory in modern function theory. - Maruzen Co., Ltd., Tokyo, 1959.

[7] Weyl, H.: Raum, Zeit, Materie. Vorlesungen über allgemeine Relativitätstheorie. - [Vierte Auflage.] Verlag von Julius Springer, Birlin, 1921.

[8] Whittaker, E. T., und G. N. Watson: A course of modern analysis. - [Vierte Auflage.] Cambridge University Press, Cambridge, 1927.

[9] Yano, K., und S. Bochner: Curvature and Betti numbers. - Annals of Mathematics Studies 32. Princeton University Press, Princeton (N. J.), 1953. 